\title{
The Miller-Rabin test with randomized exponents
}

\author{
Gebhard Böckle \\ Communicated by Igor Shparlinski
}

\begin{abstract}
We analyze a variant of the well-known Miller-Rabin test, that may be useful in preventing side-channel attacks to the random prime generation on smart cards: In the Miller-Rabin primality test for a positive integer $n$, one computes repeatedly the expression $a^{\omega}(\bmod n)$ for random bases $a \in \mathbb{N}$ and exponents $\omega$ such that $\omega$ divides $n-1$ and $(n-1) / \omega$ is a power of 2 . In each round one chooses, at random, a different base $a$, and uses binary exponentiation to compute $a^{\omega}$ $(\bmod n)$. 'Listening' to many rounds, it seems at least plausible that an outside spy could retrieve the integer $n-1$.

In the variant we consider, one chooses in each round two positive random integers $a$ and $\rho$ and applies the test with base $a$ and exponents $\omega \rho, \omega$ as above. This increases the safety against side-channel attacks. However at the same time, it decreases the performance of the Miller-Rabin test. In this article we use elementary means to analyze this variant. We will not be able to obtain results as strong as those by Damgård, Landrock and Pomerance on prime generation using the original Miller-Rabin test. However by imposing restrictions on the random parameter $\rho$, we obtain satisfactory estimates on the variant described here which justify practical implementation.
\end{abstract}

Keywords. Miller-Rabin test, secure prime generation, side channel attacks.

AMS classification. 11Y11 (94A60).

\section{Introduction}

To generate random prime numbers on smart cards, one typically uses the probabilistic primality test of Miller-Rabin. If an integer passes many rounds of this test, it is very likely that it is a prime number. If one chooses at random a $k$-bit integer $n$, and if it successfully passes $t$ rounds, then very good estimates for the probability that $n$ is prime, have been obtained by Damgård, Landrock and Pomerance in [3].

However if one implements the Miller-Rabin test straightforwardly on a smart card, then using side-channel attacks it seems possible that an outside spy can retrieve the integer $n$. The point is that in the Miller-Rabin test, one uses binary exponentiation, always with the same exponents, namely the divisors $\omega$ of $n-1$ such that $(n-1) / \omega$ is a power of 2. So if a spy can 'listen' to many rounds of the test, it seems probable that the secret $n$ is revealed. This attack was first observed in the seminal article [5] by Kocher. To avoid this kind of attack, we analyze here a variant of the Miller-Rabin test. We also refer to [2] for a wider perspective on secure prime number generation.

Let us first recall the original test for an odd integer $n$ : One writes $n-1=2^{s} w$ for an odd integer $w$ and $s \in \mathbb{N}$. Then one chooses an integer $a \in\{1,2, \ldots, n-1\}$ at 
random and tests whether one of the following conditions holds:

$$
\begin{aligned}
a^{w} & \equiv & 1 & (\bmod n), \text { or } \\
a^{2^{\sigma} w} & \equiv & -1 & (\bmod n), \text { for some } \sigma \in\{0,1, \ldots, s-1\} .
\end{aligned}
$$

Let $\alpha(n)$ denote the ratio of those integers $a$ in $\{1,2, \ldots, n-1\}$ for which the above test is successful, divided by the number of all integers $a$ in this range which are prime to $n$. If $n$ is a prime, then $\mathbb{Z}_{n}^{*}$ is cyclic of order $n-1$, and the test will be successful for all such $a$, so that $\alpha(n)=1$. If $n$ is composite, then it was shown by Rabin [7] and Monier [6] that $\alpha(n) \leq 1 / 4$. Hence for composite $n$, the ratio of those integers $a$ in $\{1,2, \ldots, n-1\}$ for which the above test is successful, divided by the number of all integers $a$ in this range, as smaller than $1 / 4$. The advantage of working with $\alpha(n)$ instead of the former ratio is that it is given by an 'explicit' formula.

The above test is then repeated a certain number of times, to improve the reliability of the outcome. To improve performance one typically takes $a=2$ in the first round, and one also verifies by elementary means that $n$ is not divisible by small primes.

Let us now describe the variant we are interested in: Again $n$ will be a random odd integer and one writes $n-1=2^{s} w$ as above. We also fix a subset $R \subseteq \mathbb{N}$ of odd integers to be specified later. Then one chooses integers $a \in\{1,2, \ldots, n-1\}$ and $\rho \in R$ at random and one computes $w \rho$. The new test verifies whether one of the following conditions holds:

$$
\begin{array}{rlrl}
a^{w \rho} & \equiv & 1 & (\bmod n), \text { or } \\
a^{2^{\sigma} w \rho} & \equiv-1 & (\bmod n), \text { for some } \sigma \in\{0,1, \ldots, s-1\} .
\end{array}
$$

For fixed $\rho$, we denote the above test by $\mathrm{MR}_{\rho}$. For a random choice $\rho \in R$, we denote it by $\mathrm{MR}_{R}$, and call it the Miller-Rabin test with randomized exponent (in $R$ ). By $\alpha_{\rho}(n)$ we denote the ratio of those $a$ for which $\mathrm{MR}_{\rho}$ is successful, divided by $\# \mathbb{Z}_{n}^{*}$, and by $\alpha_{R}(n)$ the average over the $\alpha_{\rho}(n)$ for $\rho \in R$. It is obvious from these definitions that $\alpha(n) \leq \alpha_{\rho}(n), \alpha_{R}(n)$.

We introduce some more notation: By $\pi_{i}$ we denote the $i$-th prime, so $\left(\pi_{i}\right)$ is the sequence $(2,3,5, \ldots)$, and by $P_{i}$ we denote the product of the first $i$ primes.

In the sequel, the set $R$ will always be an arithmetic progression of the following type: For $i \in \mathbb{N}, \rho_{0} \in\left\{1,2, \ldots, P_{i}-1\right\}$ which is supposed to be prime to $P_{i}$, and $r \in \mathbb{N}$ we set

$$
R_{i, \rho_{0}, r}:=\left\{\rho_{0}+\lambda P_{i}: \lambda=0,1, \ldots, r-1\right\} .
$$

In particular any element in $R_{i, \rho_{0}, r}$ is prime to the first $i$ prime numbers, and the cardinality of $R_{i, \rho_{0}, r}$ is $r$. If $i=1$, then $R_{i, \rho_{0}, r}$ is simply the set of the $r$ smallest positive odd integers.

Proposition 1.1. If $n$ is prime, then $\alpha_{R}(n)=1$ for any set $R$ (of odd integers).

If $n$ is composite, one has the following estimates:

(a) Suppose $R=R_{1,1, r}$ where (i) $18 \leq r$, (ii) $8 r^{2} \leq n$, and (iii) $r$ is divisible by 3 . Then $\alpha_{R}(n) \leq \frac{1}{4}$ unless $n$ is of the form $n=p(3 p-2)$ with $p, 3 p-2$ prime and $p \equiv-1(\bmod 4)$. In this latter case $\alpha_{R}(n)=\frac{5}{18}$. 
(b) Suppose $R=R_{i, \rho_{0}, r}$ for some $i \geq 2$ where (i') $2 \pi_{i+1}^{2} \leq r$ and (ii') $2 r^{2} P_{i} \leq n$. Then whenever $\alpha_{R}(n) \neq \alpha(n)$ one has $\alpha_{R}(n) \leq \frac{1}{\pi_{i+1}}$.

The case (a) had been previously considered by J. Gerhard, [4], who had essentially obtained the same result, except for the precise determination of the exceptional set of composite $n$ with $\alpha_{R}(n)>\frac{1}{4}$.

The integers $n$ with $\alpha_{R}(n)>\frac{1}{4}$ are precisely those with $\alpha(n)=\frac{1}{6}$. The latter set had first been classified in [3, Thm. 4 (ii)]. The factorization $n=p(3 p-2)$ easily implies that for such $n$ the number $3 n+1$ is a square. Hence the density of such $n$ of bit length $k$ is at most $2^{-k / 2}$. This estimate is used in Proposition 1.2. Numerical experiments suggest that the density of such $n$ is actually bounded by $k^{-2} 2^{2-\frac{k}{2}}$. This might indicate that the density of integers $p$ of size roughly $x$ such that $p$ as well as $3 p-2$ is a prime is $\asymp(\log (x))^{-2}$.

We now follow the usual prime generation method described for instance in [3]: For this we fix suitable parameters $i, \rho_{0}, r$ as above and set $R=R_{i, \rho_{0}, r}$. Then a $k$-bit integer $n$ is chosen at random. If $n$ passes the test $\operatorname{MR}_{\{1\}}$ with $a=2$ and $t-1$ times the test $\mathrm{MR}_{R}$, then it is declared to be prime and we stop. If not, we choose another integer $n$ at random. If it passes the test $\mathrm{MR}_{\{1\}}$ with $a=2$ and $t-1$ times the test $\mathrm{MR}_{R}$, then it is declared to be prime and we stop. This process is repeated until a (probable) prime $n$ is found. We denote the resulting algorithm, which only lets pass integers $n$ that pass tests of the form $\mathrm{MR}_{R}$ at least $t$-times, by $\mathrm{MR}_{R}^{t}$.

The reliability of the test $\mathrm{MR}_{R}^{t}$ is investigated below in Propositions 1.2 and 1.3 and in Corollary 1.4. These are the main results of the present article. The improved security to side channel attacks of $\mathrm{MR}_{R}^{t}$ in comparison with $t$ rounds of the original Miller-Rabin test lies in the fact that the exponent $w \rho$ is changed in every round. Since the value of $\rho$ is chosen at random from a rather large set $R$, the exponents $w \rho$ used in different rounds appear to an outsider as independent random choices. Suppose an outsider can gather partial knowledge on the exponents of each test. For $\mathrm{MR}_{R}^{t}$ this is information on $t$ independent exponents $w \rho$, for the original test it is $t$ times information on the same exponent. Clearly the partial information obtained in the new test is more difficult to combine. In fact, we think that this is probably very hard, but we do not analyze this here. Finally we note that the multiplication $w \cdot \rho$ is secure: While standard implementations of binary exponentiation may leak information on the exponent [5], multiplication of two large random integers does not.

To analyze the reliability of the above algorithm, we define $q_{k, t, R}$ as the probability that a composite $k$-bit integer passes the test $\operatorname{MR}_{R}^{t}$. Because $\alpha(n)$ is on the average much smaller than $\frac{1}{4}$ and because of the prime number distribution, the bounds given in [3] for $q_{k, t}:=q_{k, t,\{1\}}$ are much better than $4^{-t}$. In Section 4 we shall prove the following bounds for the algorithm based on $\mathrm{MR}_{R}$ :

Proposition 1.2. Suppose that $18 \leq r$, that 3 divides $r$ and that $r$ satisfies $8 r^{2} \leq 2^{k-1}$. Then

$$
q_{k, t, R_{1,1, r}} \leq\left(\frac{1}{4}\right)^{t-1} \frac{q_{k, 1}}{1-q_{k, 1}}+\left(\frac{5}{18}\right)^{t-1} k 2^{1-k / 2} .
$$


Proposition 1.3. Suppose that $2 \leq i, 2 \pi_{i+1}^{2} \leq r$ and $2 r^{2} P_{i} \leq 2^{k-1}$. Then

$$
q_{k, t, R_{i, \rho_{0}, r}} \leq q_{k, t}+\frac{q_{k, 1}}{1-q_{k, 1}} \pi_{i+1}^{1-t} .
$$

By specializing Proposition 1.3 and using the known values from [3, p. 194 ${ }^{1}$ for $q_{k, 1}$, it is straightforward to derive the following practical consequences.

Corollary 1.4. Let $k=512$ and $R=R_{6,1,2^{17}}$. Then $q_{k, t, R} \leq 2^{-57-4(t-1)}$.

$$
\text { Let } k=1024 \text { and } R=R_{11,1,2^{27}} \text {. Then } q_{k, t, R} \leq 2^{-150-2(t-1)} 3^{-2(t-1)} \text {. }
$$

The set $R$ in the corollary consists of 32- and 64-bit integers, respectively.

It seems likely to the present author that in fact one can prove estimates similar to those in [3] without restrictions on the set of random exponents. However a straightforward adaption of the method of [3] seems not possible. The only information used in the proof in op.cit. are a bound on the number of $n$ such that $\alpha(n)$ is above a certain size. However the randomized $\alpha_{\rho}(n)$ may be much larger than $\alpha(n)$ for a positive density of $\rho$. To adapt [3] one would therefore also need to analyze the number and size of prime factors in the prime decomposition of $p-1$ for the prime divisors $p$ of $n$.

At the same time, it seems very likely that, by analyzing a small number of exceptional cases, one can improve the base $\frac{1}{\pi_{i+1}}$ in Proposition 1.3 by $\frac{1}{2 \pi_{i+1}}$, or even further. This would yield better bounds in Corollary 1.4.

\section{A probabilistic lemma}

Fix $r, d \in \mathbb{N}$ and $b \in \mathbb{N}$ relatively prime to $d$ such that $1 \leq b<d$. Let $R \subseteq \mathbb{N}$ be the arithmetic progression $\{b+d s: 0 \leq s \leq r-1\}$ of $r$ elements.

For an integer $m$, we define $\operatorname{prob}(m$ div. $\rho \mid \rho \in R):=\mid\{\rho \in R: m$ divides $\rho\}|/| R \mid$, i.e. the probability that a random integer of $R$ is divisible by $m$.

Lemma 2.1. (a) If $m \geq d r$, then $\operatorname{prob}(m$ div. $\rho \mid \rho \in R)=0$.

(b) If $\operatorname{prob}(m \operatorname{div} . \rho \mid \rho \in R)>0$, then $\operatorname{prob}(m \operatorname{div} . \rho \mid \rho \in R)<\frac{1}{m}+\frac{1}{r}$.

(c) If $\operatorname{prob}(m \operatorname{div} . \rho \mid \rho \in R)>0$ and if $m \mid r$, then $\operatorname{prob}(m \operatorname{div} . \rho \mid \rho \in R)=\frac{1}{m}$.

Proof. Since (a) is obvious, we now turn to (b) and (c). If $\operatorname{prob}(m$ div. $\rho \mid \rho \in R)>0$ then $m$ is relatively prime to $d$, since if a prime $p$ divides $b+d s$ and $d$ at the same time, it will also divide $b$ and $d$ at the same time which is impossible since $\operatorname{gcd}(b, d)=1$.

Because $m$ is prime to $d$, the residue classes $d s(\bmod m), s=0, \ldots, m-1$, are a complete list of the elements of $\mathbb{Z} /(m)$. Since $R$ is an arithmetic progression, with steps of length $d$, it follows that the progression modulo $m$ is $m$-periodic, and not periodic for any number smaller than $m$. In particular if $r$ is a multiple of $m$, then any residue class occurs exactly $r / m$ many times. This shows (c).

For general $r$, the residue class zero modulo $m$ occurs most often if it occurs for $s=0, m, 2 m, \ldots$. If $\lambda$ denotes the number of occurrences of zero, then we must have

\footnotetext{
${ }^{1}$ The values $q_{512,1} \approx 2^{-57}$ and $q_{1024,1} \approx 2^{-150}$ are obtained by extrapolation from the table in [3].
} 
$m(\lambda-1) \leq r-1$, i.e. $\lambda \leq(r-1) / m+1$. Dividing by $r$, we find $\operatorname{prob}(m \operatorname{div} . \rho \mid \rho \in$ $R)=\lambda / r<\frac{1}{m}+\frac{1}{r}$, as asserted.

The main problem in obtaining the estimates for Proposition 1.1 will be that the set $R$ is typically relatively small compared to $n$. Thus if $q$ is a prime divisor of $n-1$ or of $p-1$ for $p$ a prime dividing $n-1$, then $\operatorname{prob}(q$ div. $\rho \mid \rho \in R)$ may differ by up to $1 /|R|$ from the expected number $\frac{1}{q}$ that is obtained for $|R| \rightarrow \infty$. The quantity $a_{R}(n)$ will by a weighted average over the probabilities $\operatorname{prob}(q \operatorname{div} . \rho \mid \rho \in R)$, with possibly many summands. Therefore the 'error terms' $1 /|R|$ may sum up to a significant error term in the sum, if one is not careful. The following simple probabilistic lemma will be used to show that indeed the error terms do not add up.

Let us introduce some notation. Let $S:=\{1,2, \ldots, h\}$ be a subset of consecutive integers. Let $\mu$ be a probability measure on the power set $\{0,1\}^{S}$ of $S$, i.e., we regard the subsets of $S$ as points of some space and attach to each such a point mass. Fix some function $g: S \rightarrow \mathbb{R}_{>0}: j \mapsto g_{j}$ and abbreviate $g_{J}:=\prod_{j \in J} g_{j}$ for any $J \subseteq S$ (note that whenever a product is formed over the empty set as an index set, the value of the product is supposed to be one). We define the $g$-weighted average over $\mu$ as

$$
\alpha(g, \mu):=\sum_{J \subseteq S} \mu(J) g_{S \backslash J} .
$$

Lemma 2.2. With the notation as above one has

$$
\alpha(g, \mu)=\sum_{J \subseteq S} g_{S \backslash J}\left(\sum_{K \supseteq J} \mu(K)\right)\left(\sum_{L \subseteq J}(-1)^{|L|} g_{L}\right) .
$$

Lemma 2.2 may be regarded as a multi-dimensional version of Abel summation, cf. (AS) on page 315 .

Proof. The proof is given by rewriting and simplifying the right hand side:

$$
\begin{aligned}
\sum_{J \subseteq S} \sum_{K \supseteq J} \sum_{L \subseteq J} g_{S \backslash J} \mu(K)(-1)^{|L|} g_{L} & \\
= & \sum_{K \subseteq S} \mu(K) \sum_{L \subseteq J \subseteq K} g_{S \backslash J}(-1)^{|L|} g_{L} \\
= & \sum_{K \subseteq S} \mu(K) g_{S \backslash K} \sum_{L \subseteq J \subseteq K}(-1)^{|L|} g_{(K \backslash J) \cup L} \\
M=(K \backslash J) \cup L & \sum_{K \subseteq S} \mu(K) g_{S \backslash K} \sum_{M \subseteq K} g_{M} \sum_{L \subseteq M}(-1)^{|L|} \\
= & \sum_{K \subseteq S} \mu(K) g_{S \backslash K} \sum_{M=\varnothing} g_{M} \sum_{L \subseteq M}(-1)^{|L|}=\sum_{K \subseteq S} \mu(K) g_{S \backslash K},
\end{aligned}
$$

where in the last line we use $\sum_{L \subseteq M}(-1)^{|L|}=0$ for $M \neq \varnothing$. 
The above lemma will be applied in the following situation:

We will consider $h$ distinct prime numbers $q_{1}, \ldots, q_{h}$. For each there is an exponent $f_{j} \in \mathbb{N}$, and we will set $g_{j}:=q_{j}^{-f_{j}}$. Moreover we will consider the following three probability measures:

$$
\begin{aligned}
\mu_{0}(J):=\operatorname{prob}\left(\left(\prod_{j \in J} q_{j} \text { div. } \rho\right) \text { and }\left(\prod_{j \notin J} q_{j} \text { is prime to } \rho\right) \mid \rho \in R\right) . \\
\mu_{1}(J):=\prod_{j \in J} q_{j}^{-1} \prod_{j \notin J}\left(1-q_{j}^{-1}\right) . \\
\mu_{2}(J):= \begin{cases}0, & \text { if } J \neq S, \\
1, & \text { if } J=S .\end{cases}
\end{aligned}
$$

The measure $\mu_{1}$ is so to speak the limit of $\mu_{0}$ as $r \rightarrow \infty$ (for a random set $R$ ). Note that for fixed $J \subseteq S$ we have the following expressions for $\sum_{K \supseteq J} \mu_{i}(K)$ :

$$
\sum_{K \supseteq J} \mu_{i}(K)=\left\{\begin{array}{cl}
\operatorname{prob}\left(\prod_{j \in J} q_{j} \operatorname{div} . \rho \mid \rho \in R\right) \stackrel{2.1(\mathrm{~b})}{\leq} \prod_{j \in J} q_{j}^{-1}+\frac{1}{r} & \text { for } i=0, \\
\prod_{j \in J} q_{j}^{-1} & \text { for } i=1, \\
1 & \text { for } i=2 .
\end{array}\right.
$$

\section{A single round of the Miller-Rabin variant}

This section is mainly concerned with the proof of Proposition 1.1. Throughout this section we fix an odd integer $n \geq 3$ and write $n=p_{1}^{e_{1}} \cdots p_{\ell}^{e_{\ell}}$ for its factorization into distinct prime powers. We define odd integers $w$ and $w_{i}$ and integers $s$ and $s_{i}$ such that $2^{s} w=n-1$ and $2^{s_{i}} w_{i}=p_{i}-1$. For $x \in \mathbb{N}$ we set

$$
\gamma(x):=\prod_{i=1}^{\ell} \frac{\operatorname{gcd}\left(w_{i} p_{i}^{e_{i}-1}, x\right)}{w_{i} p_{i}^{e_{i}-1}} .
$$

We also set $\underline{s}:=\min \left\{s_{i}: i=1, \ldots, \ell\right\}$, and define

$$
\beta(n):=2^{-\sum_{i} s_{i}}\left(1+\frac{2^{l \underline{s}}-1}{2^{\ell}-1}\right) .
$$

Recall that by $\alpha_{\rho}(n)$ we denote the ratio of the number of those $a \in\{1, \ldots, n\}$ for which the variant $\mathrm{MR}_{\rho}$ of the Miller-Rabin test is successful, divided by $\mathbb{\#} \mathbb{Z}_{n}^{*}$.

Lemma 3.1. One has $\alpha_{\rho}(n)=\beta(n) \gamma(\rho(n-1))$ and $\beta(n) \leq 2^{1-\ell}$.

Proof. We consider the sets $G_{0}:=\left\{a \in \mathbb{Z}_{n}^{*}: a^{w \rho}=1\right\}$, and

$$
G_{j}:=\left\{a \in \mathbb{Z}_{n}^{*}: a^{2^{j-1} w \rho}=-1\right\}
$$


for $j=1, \ldots, s$. By the Chinese remainder we have

$$
\mathbb{Z}_{n}^{*} \cong \mathbb{Z}_{p_{1}}^{*} \times \cdots \times \mathbb{Z}_{p_{\ell}^{e_{1}}}^{*} \times
$$

Since the $p_{i}$ are all odd, the factors on the right are all cyclic. The elements \pm 1 in $\mathbb{Z}_{n}$ have component \pm 1 in each of the rings $\mathbb{Z}_{p_{i} e_{i}}$, and the sets $G_{j}$ are products of corresponding sets for each $i=1, \ldots, \ell$.

Because $w \rho$ is odd and $\mathbb{Z}_{p_{i}^{e_{i}}}^{*}$ is cyclic of order $2^{s_{i}} w_{i} p_{i}^{e_{i}-1}$, it is easy to see that for each $i, j$

$$
\left|\left\{a \in \mathbb{Z}_{p_{i}^{e_{i}}}^{*}: a^{2^{j-1} w \rho}=-1\right\}\right|=\left\{\begin{array}{cl}
2^{j-1} \operatorname{gcd}\left(w \rho, w_{i} p_{i}^{e_{i}-1}\right) & \text { for } j \leq s_{i} \\
0 & \text { otherwise }
\end{array}\right.
$$

and moreover $\left|\left\{a \in \mathbb{Z}_{p_{i} e_{i}}: a^{w \rho}=1\right\}\right|=\operatorname{gcd}\left(w \rho, w_{i} p_{i}^{e_{i}-1}\right)$. Combining these results for fixed $j$ and all the $i$, we find

$$
\left|G_{j}\right|=\left\{\begin{array}{cc}
2^{\ell \max \{0, j-1\}} \prod_{i=1}^{\ell} \operatorname{gcd}\left(w \rho, w_{i} p_{i}^{e_{i}-1}\right) & \text { for } j \leq \underline{s} \\
0 & \text { otherwise }
\end{array}\right.
$$

Thus, summing over all $j$ we have

$$
\sum_{j=0}^{s}\left|G_{j}\right|=\left(1+\sum_{j=0}^{\underline{s}-1} 2^{\ell j}\right) \prod_{i=1}^{\ell} \operatorname{gcd}\left(w \rho, w_{i} p_{i}^{e_{i}-1}\right) .
$$

Dividing by the order of $\mathbb{Z}_{n}^{*}$, i.e. by $\prod_{i=1}^{\ell} 2^{s_{i}} w_{i} p_{i}^{e_{i}-1}$, yields the desired result.

Finally, by looking at the 2-adic expansion of $1+\sum_{j=0}^{s-1} 2^{\ell j}$ one finds that this sum is at most $2^{\ell(\underline{s}-1)+1}$. Because $\underline{s} \leq s_{i}$ for all $i$, the asserted bound on $\beta(n)$ follows.

We fix a set $R$ which as usual is an arithmetic progression. From its definition, it is clear that $\gamma(n-1)^{-1}$ is an odd integer. Therefore we can write

$$
\gamma(n-1)^{-1}=q_{0} \prod_{j=1, \ldots, h} q_{j}^{f_{j}}
$$

such that $q_{0}$ is an odd integer relatively prime to $\prod_{\rho \in R} \rho$, such that $q_{1}, \ldots, q_{h}$ are those (distinct) prime divisors of $\gamma(n-1)^{-1}$ which divide $\prod_{\rho \in R} \rho$, and where $f_{j} \in \mathbb{N}$ is the order of $q_{j}$ as a divisor of $\gamma(n-1)^{-1}$. Define $S:=\{1,2, \ldots, h\}$. To apply the lemma of the previous section in the proof of Proposition 1.1, we first rewrite $\alpha_{R}(n)$ in terms of the measure $\mu_{0}$ from the defining equation (2.1):

Lemma 3.2. One has

$$
\alpha_{R}(n) \leq \beta(n) \frac{1}{q_{0}} \cdot \sum_{J \subseteq S} \mu_{0}(J) \prod_{j \notin J} q_{j}^{-f_{j}} .
$$


Proof. For $J \subseteq S$, we define $R_{J}:=\left\{\rho \in R: q_{j} \mid \rho \Leftrightarrow j \in J\right\}$. Then

$\frac{\alpha_{R}(n)}{\beta(n)} \stackrel{\text { by def. }}{=} \frac{1}{|R|} \sum_{\rho \in R} \frac{\alpha_{\rho}(n)}{\beta(n)} \stackrel{\text { Lem. }}{=}{ }^{3.1} \frac{1}{|R|} \sum_{\rho \in R} \gamma(\rho(n-1))=\sum_{J \subseteq S} \frac{1}{|R|} \sum_{\rho \in R_{J}} \gamma(\rho(n-1))$.

From the defining equation (3.1) for $\gamma(x)$ and the above product representing $\gamma(n-1)^{-1}$, we have for any $\rho \in R_{J}$ the inequality

$$
\gamma(\rho(n-1)) \leq q_{0}^{-1} \prod_{j \notin J} q_{j}^{-f_{j}} .
$$

Substituting this and $\mu_{0}(J)=\left|R_{J}\right| /|R|$ in the above expression for $\alpha_{R}(n)$, we find

$$
\frac{\alpha_{R}(n)}{\beta(n)} \leq \sum_{J \subseteq S} \mu_{0}(J) q_{0}^{-1} \prod_{j \notin J} q_{j}^{-f_{j}} .
$$

Remark 3.3. (a) If $S$ is empty, then $\gamma(n-1)=q_{0}^{-1}=\gamma(\rho(n-1))$ for all $\rho \in R$, and so $\alpha_{R}(n)=\alpha(n)$.

(b) If for a given $R$ the product $\prod_{\rho \in R} \rho$ is prime to $\gamma(n-1)^{-1}$, then $S$ is empty.

(c) If $S=\left\{q_{1}\right\}$ consists of a single element, $f_{1}=1$ and $q_{1} \mid r$, then, arguing as in the previous proof, one obtains $\alpha_{R}(n)=\beta(n) \frac{1}{q_{0} q_{1}}\left(1-\frac{1}{q_{1}}\right)$.

Lemma 3.4. One has

$$
\frac{\alpha_{R}(n) q_{0}}{\beta(n)} \leq \frac{1}{r}+\prod_{j \in S} q_{j}^{-1} \sum_{J \subseteq S} \prod_{j \notin J}\left(q_{j}^{1-f_{j}}-q_{j}^{-f_{j}}\right) \leq \frac{1}{r}+\prod_{j \in S} \frac{2}{q_{j}} .
$$

Proof. The inequality on the right follows, since all the expressions $q_{j}^{1-f_{j}}-q_{j}^{-f_{j}}$ are smaller than 1 , and since $\sum_{J \subseteq S} 1=2^{|S|}$. We prove the inequality on the left:

$$
\begin{array}{rll}
\frac{\alpha_{R}(n) q_{0}}{\beta(n)} \stackrel{\stackrel{\text { Lem.3.2 }}{\leq}}{\leq} \sum_{J \subseteq S} \mu_{0}(J) \prod_{j \notin J} q_{j}^{-f_{j}} \\
\stackrel{\text { Lem. 2.2 }}{=} \sum_{J \subseteq S}\left(\prod_{j \notin J} q_{j}^{-f_{j}}\right) \sum_{K \supseteq J} \mu_{0}(K)\left(\sum_{L \subseteq J}(-1)^{|L|} \prod_{j \in L} q_{j}^{f_{j}}\right) .
\end{array}
$$

Applying all three parts of formula (2.4) we find

$$
\sum_{K \supseteq J} \mu_{0}(K) \leq \frac{1}{r}+\prod_{j \in J} q_{j}^{-1}=\frac{1}{r}\left(\sum_{K \supseteq J} \mu_{2}(K)\right)+\sum_{K \supseteq J} \mu_{1}(K) .
$$


Thus

$$
\begin{aligned}
\frac{\alpha_{R}(n) q_{0}}{\beta(n)} \leq \quad & \frac{1}{r} \sum_{J \subseteq S}\left(\prod_{j \notin J} q_{j}^{-f_{j}}\right) \sum_{K \supseteq J} \mu_{2}(K)\left(\sum_{L \subseteq J}(-1)^{|L|} \prod_{j \in L} q_{j}^{f_{j}}\right) \\
& +\sum_{J \subseteq S}\left(\prod_{j \notin J} q_{j}^{-f_{j}}\right) \sum_{K \supseteq J} \mu_{1}(K)\left(\sum_{L \subseteq J}(-1)^{|L|} \prod_{j \in L} q_{j}^{f_{j}}\right) \\
\stackrel{\text { Lem.2.2 }}{=} & \frac{1}{r} \sum_{J \subseteq S} \mu_{2}(J) \prod_{j \notin J} q_{j}^{-f_{j}}+\sum_{J \subseteq S} \mu_{1}(J) \prod_{j \notin J} q_{j}^{-f_{j}} .
\end{aligned}
$$

Since $\mu_{2}(J)=0$ for $J \neq S$, the first sum adds up to one. In the second sum, we substitute the definition of $\mu_{1}(J)$, and obtain

$$
\frac{\alpha_{R}(n) q_{0}}{\beta(n)} \leq \frac{1}{r}+\sum_{J \subseteq S} \prod_{j \in J} q_{j}^{-1} \prod_{j \notin J}\left(1-q_{j}^{-1}\right) \prod_{j \notin J} q_{j}^{-f_{j}} .
$$

Pulling out the factor $\prod_{j \in S} q_{j}^{-1}$ from the sum, yields the desired estimate.

For arbitrary $n$ (and $S \neq \varnothing$ ), the estimates in inequality (3.4) and in Lemma 3.4 can not be improved. However, if $e_{i} \geq 2$ for some factor $p_{i}^{e_{i}}$ of $n$, then better bounds can be obtained. The proof of Proposition 1.1 will need such an improved estimate in one particular case, which we now derive:

Lemma 3.5. As before, let $d$ be the width of the arithmetic progression $R$ of length $r$. Suppose that $n=p^{e}$ is a prime power and that $n>d r$. If $p \geq d r$, then $\alpha_{R}(n)=\alpha(n)$, else

$$
\alpha_{R}(n) \leq \frac{1+(e-1)\left(1-p^{-1}\right)}{p^{e-1}}+\frac{1-p^{1-e}}{r} .
$$

Proof. In the situation at hand we have $\beta(n)=1$ and $\gamma(n-1)=p^{1-e}$, and therefore $\alpha(n)=p^{1-e}$ as well. Note that for $n=p$ one has $\alpha(n)=\alpha_{R}(n)=1$ and the right hand side of equation (3.5) is 1 as well. Thus from now on, we assume $e \geq 2$.

We first consider the case where $p$ is not a divisor of $\prod_{\rho \in R} \rho$. Then $\gamma(n-1)^{-1}$ is prime to $\prod_{\rho \in R} \rho$, and so $\alpha_{R}(n)=\alpha(n)=p^{1-e}$ by Remark 3.3. If $p \geq d r$, i.e., if $p$ is larger than the largest element of the arithmetic expression $R$, then $\alpha_{R}(n)=\alpha(n)$, as asserted. If $p<d r$ (and still $p$ does not divide any of the $\rho \in R$ ), then equation (3.5) holds because the right hand side of equation (3.5) is larger than $p^{1-e}$, as $e \geq 2$.

From now on, we assume that $p$ divides one of the $\rho \in R$. In formula (3.3) we have $q_{0}=1, h=1, q_{1}=p$ and $f_{1}=e-1$. Clearly now $p<d r$, and so we need to prove equation (3.5).

The key point in the following chain of inequalities is Abel summation

$$
\sum_{i=0}^{g}\left(a_{i}-a_{i+1}\right) b_{i}=a_{0} b_{0}+\sum_{i=1}^{g} a_{i}\left(b_{i}-b_{i-1}\right)-a_{g+1} b_{g}
$$


and the observation that in the case at hand the defining formula (3.1) simplifies to $\gamma(\rho(n-1))=\frac{\operatorname{gcd}\left(p^{e-1}, \rho\right)}{p^{e-1}}$ :

$$
\begin{aligned}
& \alpha_{R}(n)=\frac{1}{r} \sum_{\rho \in R} \alpha_{\rho}(n) \stackrel{\text { Lem. }}{=} .1 \frac{1}{r} \sum_{\rho \in R} \gamma(\rho(n-1)) \\
& \stackrel{(3.1)}{=} \sum_{t=0}^{e-1}\left(\operatorname{prob}\left(p^{t} \operatorname{div} . \rho \mid \rho \in R\right)-\operatorname{prob}\left(p^{t+1} \operatorname{div} . \rho \mid \rho \in R\right)\right) p^{1-e+t} \\
& \stackrel{(\mathrm{AS})}{=} p^{1-e}+\sum_{t=1}^{e-1} \operatorname{prob}\left(p^{t} \operatorname{div} . \rho \mid \rho \in R\right)\left(p^{1-e+t}-p^{-e+t}\right)-\operatorname{prob}\left(p^{e} \operatorname{div} . \rho \mid \rho \in R\right) .
\end{aligned}
$$

To obtain an upper bound we may drop the last term and estimate the remaining summands using Lemma 2.1. This implies

$$
\alpha_{R}(n) \leq p^{1-e}+\sum_{t=1}^{e-1}\left(p^{-t}+\frac{1}{r}\right)\left(p^{1-e+t}-p^{-e+t}\right) .
$$

The terms involving $\frac{1}{r}$ form a telescoping sum, the other ones a sum over constants. Evaluation of the expressions now yields the estimate (3.5).

Proof of Proposition 1.1. From now on, we take $R:=R_{i, \rho_{0}, r}$, so that $R$ is an arithmetic progression of length $r$ and width $P_{i}$. We first consider the case where $n$ is a power $p^{e}$ of some prime $p$ but not a prime number. In this case $\alpha(n)$ and $\alpha_{R}(n)$ are typically very small, provided in case (b) that the elements in $R$ are small compared to $n$ : Note first that by Remark 3.3 for $p \geq P_{i} r$ we have $\alpha(n)=\alpha_{R}(n)$, so that we may assume $p<$ $P_{i} r$. Since $P_{i} \geq 2$, we have $\ln \left(P_{i} r\right) \geq \ln (2 r)$, and hence also $2 \ln \left(P_{i} r\right) \geq \ln \left(2 P_{i} r^{2}\right)$. Because $n \geq 2 P_{i} r^{2}$ and $x \mapsto \frac{x}{\ln (x)}$ is strictly increasing for $x>\exp (1)$, this shows

$$
\frac{n}{\ln (n)} \geq \frac{2 P_{i} r^{2}}{\ln \left(2 P_{i} r^{2}\right)} \geq \frac{P_{i} r}{\ln \left(P_{i} r\right)} r
$$

Since $P_{i} r>p \geq 3$, the previous inequality yields

$$
n \geq \frac{\ln (n)}{\ln (p)} p r
$$

which is equivalent to $n \geq e p r$, or to $\frac{p^{e-1}}{e} \geq r$. Hence our hypotheses yield

$$
\alpha_{R}(n) \stackrel{\text { Lem. } 3.5}{\leq} \frac{1+(e-1)\left(1-p^{-1}\right)}{p^{e-1}}+\frac{1-p^{-e}}{r} \leq \frac{e}{p^{e-1}}+\frac{1}{r} \leq \frac{2}{r} \leq \frac{1}{2 \pi_{i+1}},
$$

where for the last inequality we use $r \geq 2 \pi_{i+1}^{2}$.

Next we consider the case where $n$ has at least three prime factors, or where $n$ has two prime factors and $s_{1} \neq s_{2}$. In either case, by Lemma 3.1 or the by defining 
expression (3.2) one finds $\beta(n) \leq \frac{1}{4}$. If $S=\varnothing$, then $\alpha_{R}(n)=\alpha(n)$ and there is nothing to prove. If $|S|=2$, then Lemma 3.4 yields

$$
\alpha_{R}(n) \leq \frac{1}{4}\left(\frac{1}{r}+\frac{4}{\pi_{i+1} \pi_{i+2}}\right) \leq \frac{1}{2 \pi_{i+1}},
$$

because $\rho$ has no prime divisor smaller than $\pi_{i+1}$ and $r \geq 2 \pi_{i+1}^{2}$. If $|S|=1$, then again from Lemma 3.4 we obtain

$$
\alpha_{R}(n) \leq \frac{1}{4}\left(\frac{1}{r}+\frac{1}{\pi_{i+1}}\left(2-\frac{1}{\pi_{i+1}}\right)\right) \leq \frac{1}{2 \pi_{i+1}},
$$

using the same hypothesis $r \geq 2 \pi_{i+1}^{2}$.

Finally, we assume that $n=p_{1}^{e_{1}} p_{2}^{e_{2}}$ has exactly two prime factors and that $s_{1}=s_{2}$. Then for $s_{1}=1$ one has $\beta(n)=\frac{1}{2}$ and for $s_{1} \geq 2$ one has $\beta(n) \leq \frac{3}{8}$. As in the previous paragraph $S=\varnothing$ leads to $\alpha(n)=\alpha_{R}(n)$ and $|S| \geq 2$ leads to

$$
\alpha_{R}(n) \leq \frac{1}{2}\left(\frac{1}{r}+\frac{4}{\pi_{i+1} \pi_{i+2}}\right) .
$$

In case (a) of Proposition 1.1 one finds the estimate $\alpha_{R}(n) \leq \frac{29}{180}<\frac{1}{4}$, in case (b) the estimate $\alpha_{R}(n) \leq \frac{1}{2 \pi_{i+1}}$, and in either case the proposition is proved.

It remains to consider the case $|S|=1$. In case (b) the estimate

$$
\alpha_{R}(n) \leq \frac{1}{2}\left(\frac{1}{r}+\frac{1}{\pi_{i+1}}\left(2-\frac{1}{\pi_{i+1}}\right)\right)
$$

yields, by the same arguments as above, that $\alpha_{R}(n) \leq \frac{1}{\pi_{i+1}}$.

In case (a) a direct application of Lemma 3.2 provides the estimate

$$
\alpha_{R}(n) \leq \frac{\beta(n)}{q_{0}}\left(q_{1}^{-f_{1}}+\operatorname{prob}\left(q_{1} \text { div. } \rho \mid \rho \in R\right)\left(1-q_{1}^{-f_{1}}\right)\right) .
$$

Using the above bound on $\beta(n)$, the fact that $q_{0}$ is an odd natural number and that $q_{1}$ is an odd prime, and that $\operatorname{prob}\left(q_{1}\right.$ div. $\left.\rho \mid \rho \in R\right) \leq \frac{1}{r}+\frac{1}{q_{1}}$, a short calculation proves the following: Unless $q_{1}=3, q_{0}=1$ and $\beta(n)=\frac{1}{2}$, the quantity $\alpha_{R}(n)$ is bounded above by $\frac{1}{4}$. In the exceptional case, one computes $\alpha_{R}(n)=\frac{5}{18}$ using Remark 3.3(c) which applies due to our hypothesis that 3 divides $r$. One easily verifies that the set of exceptional composite $n$ described in Proposition 1.1(a) all satisfy $\alpha_{R}(n)=\frac{5}{18}$.

To complete the proof, we need to characterize those $n$ for which $\alpha_{R}(n)=\frac{5}{18}$ can occur: By the above we must have $\beta(n)=\frac{1}{2}$ and $\gamma(n-1)^{-1}=3$, and thus $\alpha(n)=\frac{1}{6}$. The corresponding $n$ are classified in [3, Theorem 4] case (ii). As noted after the statement of Proposition 1.1 these are precisely the exceptional $n$ from part (a).

\section{Prime generation using the Miller-Rabin variant}

We now prove Proposition 1.3. The method is taken from [1], and is also present in [4]; but might go back at least to [3]. 
Proof of Proposition 1.3. Let $\sum^{\prime}$ denote the sum over all composite integers in a given range and let $M_{k}$ denote the set of all $k$-bit integers. Then

$$
\begin{aligned}
& q_{k, t, R}=\frac{\text { the probability that a composite } k \text {-bit integer passes } \mathrm{MR}_{R}^{t}}{\text { the probability that a } k \text {-bit integer passes } \mathrm{MR}_{R}^{t}} \\
& =\frac{\sum_{n \in M_{k}}^{\prime} \alpha(n) \alpha_{R}(n)^{t-1}}{\sum_{n \in M_{k}} \alpha(n) \alpha_{R}(n)^{t-1}}
\end{aligned}
$$

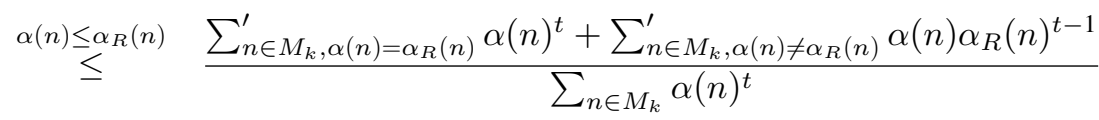

$$
\begin{aligned}
& \stackrel{\text { Prop. 1.1(b) }}{\leq} \quad q_{k, t}+\pi_{i+1}^{1-t} \frac{\sum_{n \in M_{k}, \alpha(n) \neq \alpha_{R}(n)}^{\prime} \alpha(n)}{\sum_{n \in M_{k}, n \text { prime }} 1} \\
& \leq \quad q_{k, t}+\pi_{i+1}^{1-t} \frac{\sum_{n \in M_{k}}^{\prime} \alpha(n)}{\sum_{n \in M_{k}} \alpha(n)-\sum_{n \in M_{k}}^{\prime} \alpha(n)} \\
& =\quad q_{k, t}+\pi_{i+1}^{1-t} \frac{q_{k, 1}}{1-q_{k, 1}} .
\end{aligned}
$$

In a similar way, however using directly the density of squares and of primes of bit length $k$, one may derive Proposition 1.2. We omit details.

Acknowledgments. I would like to express many thanks to the technical support group of cryptovision who brought the above problem to my attention, and gave me access to the unpublished work [4] of J. Gerhard who had first analyzed the above randomization algorithm for $i=1$ systematically. Also thanks to $\mathrm{S}$. Wentzig for a very careful reading of a preliminary version and many suggestions to improve the readability of the manuscript. Finally I want to thank the referee whose many comments further improved the readability of the present article. During the final preparation of the manuscript, the author was supported by the DFG as a member of the SFB-TR 45.

\section{References}

[1] R. J. Burthe, Further Investigations with the Strong Probable Prime Test, Math. Comp. 65 (1996), 373-381.

[2] H. Chabanne, E. Dotton, L. Ramsamy, Masked Prime Number Generation, First Benelux Workshop on Information and System Security (2006), article available under http://www.cosic.esat.kuleuven.be/wissec2006/papers/29.pdf.

[3] I. Damgård, P. Landrock, C. Pomerance, Average Case Error Estimates for the Strong Probable Prime Test, Math. Comp. 61 (1993), 177-194.

[4] J. Gerhard, Randomizing the Exponent in the Miller-Rabin Test, preprint, Dec. 10, 2002.

[5] P. C. Kocher, Attacks on Implementations of Diffie-Hellmann, RSA, DSS and Other Systems, LNCS 1109 (1996), 104-113. 
[6] L. Monier, Evaluation and comparison of two efficient probabilistic primality testing algorithms, Theoret. Comp. Sci. 12 (1980), 97-108.

[7] M. O. Rabin, Probabilistic Algorithms for Testing Primality, J. Number Theory 12 (1980), 128-138.

Received 19 February, 2009; revised 15 November, 2009

\section{Author information}

Gebhard Böckle, Fakultät für Mathematik, Universität Duisburg-Essen, Campus Essen,

45117 Essen, Germany.

Email: gebhard.boeckleduni-due.de 